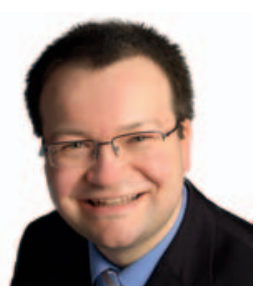

Marek Lommatzsch

marek.lommatzsch@med.uni-rostock.de

\title{
Neue Horizonte in der Prävention, Diagnostik und Therapie von Asthma
}

sition dar, welche zur nachhaltigen Veränderung der Immunantwort gegenüber Allergenen führt. Die immense Chance, die eine rechtzeitige Behandlung der allergischen Rhinitis mittels spezifischer Immuntherapie zur Asthmaprävention bietet, ist in den letzten Jahren noch klarer geworden $[6,7]$. Umso erschreckender ist, dass die spezifische Immuntherapie in Deutschland immer seltener zur Anwendung kommt [1].

\section{Asthmadiagnostik - mehr als die Summe ihrer Teile}

In der Asthmadiagnostik ist, neben der weiterhin unverzichtbaren genauen Anamnese-Erhebung, die Erfassung bestimmter Biomarker für die Asthmaphänotypisierung in den Vordergrund gerückt [8]. Hierzu zählt die Bestimmung der BluteosinophilenKonzentration mittels Differentialblutbild, einem seit mehr als 100 Jahren bekannten Asthmamarker [9], der durch diverse aktuelle Studien mit Biologika und durch seine Kostengünstigkeit, Robustheit und allgemeine Verfügbarkeit eine Renaissance erlebt hat [3]. Des Weiteren wird die Bedeutung der Reversibilitätsprüfung der Atemwegsobstruktion für die Diagnosestellung von Asthma in den aktuellen internationalen Empfehlungen (www.ginasthma.com) zwar stärker betont, andererseits gibt es aber zunehmende Hinweise darauf, dass auch Patienten mit chronisch-obstruktiver Lungenerkrankung (COPD) teils eine erhebliche Reversibilität der Atemwegsobstruktion zeigen [10]. Dadurch ist noch deutlicher geworden, dass die Diagnosestellung von Asthma nicht durch einen einzigen Biomarker möglich ist, sondern dass es weiterhin einer sorgfältigen Synopsis verschiedener Befunde und der Anamnese durch einen geschulten Arzt bedarf [3]. Dies führt im aktuellen Zeitgeist der Fallzahlsteigerungen und Personalkürzungen in der Medizin naturgemäß zum Konflikt. Die Antwort auf diesen Konflikt kann aber nicht die Verwässerung oder künstliche Simplifizierung der Diagnose Asthma sein. Vielmehr müssen die medizinischen Entscheidungsträger auf die Unverzichtbarkeit der ärztlichen Kompetenz und Erfahrung bei der Diagnose Asthma immer wieder hingewiesen werden.

\section{Neue Horizonte für die Behandlung von Asthma}

In der Therapie von Asthma haben in den letzten Jahren viele neue Therapieoptionen den klinischen Alltag erreicht [4]. Die spezifische Immuntherapie, welche bislang nur für kontrolliertes Asthma zugelassen war, erscheint nun auch für Patienten mit nicht gut kontrolliertem Asthma als sichere Therapieoption möglich: Dies zeigt eine aktuelle Studie zur Wirksamkeit einer sublingualen Immuntherapie bei Patienten mit Asthma und Hausstaubmilbenallergie [11, 12]. Die Therapie mit dem Anticholinergikum Tiotropium ist als Zusatzoption zu einer Therapie mit inhalativen Steroiden und langwirksamen Beta-Mimetika in Deutschland im Jahr 2014 für Patienten mit Asthma stellt eine Form der gezielten Allergenexpo-

\section{KARGER}

Fax +497614520714 information@karger.com www.karger.com
(๑) 2016 S. Karger GmbH, Freiburg

Accessible online at: www.karger.com/kkp
Prof. Dr. Marek Lommatzsch Sprecher des Deutschen Lungentages

Zentrum für Innere Medizin, Abteilung Pneumologie, Universität Rostock Ernst-Heydemann-Straße 6, 18057 Rostock, Deutschland 
zugelassen worden. Für die Behandlung des schweren, therapierefraktären Asthma sind in Deutschland mittlerweile zwei Biologika zugelassen: Omalizumab (Anti-Immunglobulin-E) und Mepolizumab (Anti-Interleukin-5), welche nicht nur zu einer Reduktion der Exazerbationsrate, sondern insbesondere auch zu einer Reduktion oder sogar vollständigen Vermeidung der nebenwirkungsreichen Prednisolontherapie führen [13, 14]. Weitere Biologika, wie z.B. Dupilumab (Anti-Interleukin-4/13, welches auch die atopische Dermatitis positiv beeinflusst), stehen kurz vor der Zulassung [14] Somit schauen wir heute in eine Asthma-Therapie-Landschaft, die bei weitem nicht mehr auf eine Therapie mit inhalativen Steroiden reduziert werden kann: Die Immuntherapie gewinnt wieder an Bedeutung, die inhalative Therapie ist durch verschiedene pharmakologische Angriffspunkte und viele verschiedene Inhalationssysteme breiter aufgestellt denn je, und die gezielte Biologikatherapie ist für bestimmte Patienten eine real verfügbare und segensreiche Therapieoption.

Es wird also seit einigen Jahren immer deutlicher, dass das Forschungsthema Asthma auch in der Ära der inhalativen Steroide keineswegs an Aktualität und Spannung verloren hat. Vielmehr befinden wir uns in den frühen Morgenstunden einer Zeitenwende, in denen wir beginnen, die Prävention, Diagnostik und Therapie dieser Volkserkrankung neu zu definieren.

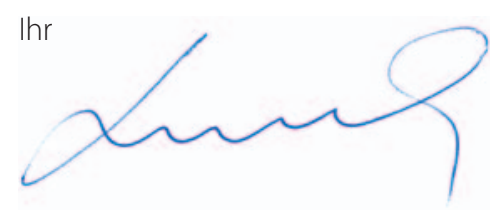

\section{Referenzen}

1 Biermann J, Merk HF, Wehrmann W, Klimek L, Wasem J: Allergische Erkrankungen der Atemwege - Ergebnisse einer umfassenden Patientenkohorte in der deutschen gesetzlichen Krankenversicherung. Allergo J 2013; 22:366-373.

2 Heederik D, von Mutius E: Does diversity of environmental microbial exposure matter for the occurrence of allergy and asthma? J Allergy Clin Immunol 2012;130:44-50.

3 Lommatzsch M: Asthma bronchiale: Es gibt kein «Asthma-HbA 1c». Dtsch Arztebl 2015;112:4.

4 Lommatzsch M, Stoll P: Novel strategies for the treatment of asthma. Allergo J Int 2016;25:11-17.

5 Du Toit G, Roberts G, Sayre PH, et al.: Randomized trial of peanut consumption in infants at risk for peanut allergy. N Engl J Med 2015;372: $803-813$.

6 Niggemann B, Jacobsen L, Dreborg S, et al.: Five-year follow-up on the PAT study: specific immunotherapy and long-term prevention of asthma in children. Allergy 2006;61:855-859.

7 Calderon MA, Kleine-Tebbe J, Linneberg A, et al.: House dust mite respiratory allergy: an overview of current therapeutic strategies. J Allergy Clin Immunol Pract 2015;3:843-855.

8 Lommatzsch M: Aktuelles in der Asthma-Diagnostik. Der Pneumologe 2013;10:394-399.

9 Rackemann FM: A clinical study of one hundred and fifty cases of bronchial asthma. Arch Intern Med 1918;12:517-552.

10 Tashkin DP, Celli B, Decramer M, et al.: Bronchodilator responsiveness in patients with COPD. Eur Respir J 2008;31:742-750.

11 Virchow J, Backer V, Kuna P, et al.: Efficacy of a house dust mite sublingual allergen immunotherapy tablet in adults with allergic asthma: a randomized clinical trial. JAMA 2016;315:1715-1725.

12 Wood RA: New horizons in allergen immunotherapy. JAMA 2016;315: 1711-1712.

13 Sweeney J, Patterson CC, Menzies-Gow A, et al.: Comorbidity in severe asthma requiring systemic corticosteroid therapy: cross-sectional data from the Optimum Patient Care Research Database and the British Thoracic Difficult Asthma Registry. Thorax 2016;71:339-346.

14 Lommatzsch M: Therapie des refraktären Asthmas mit Antikörpern. Dtsch Med Wochenschr 2016;141:790-793. 